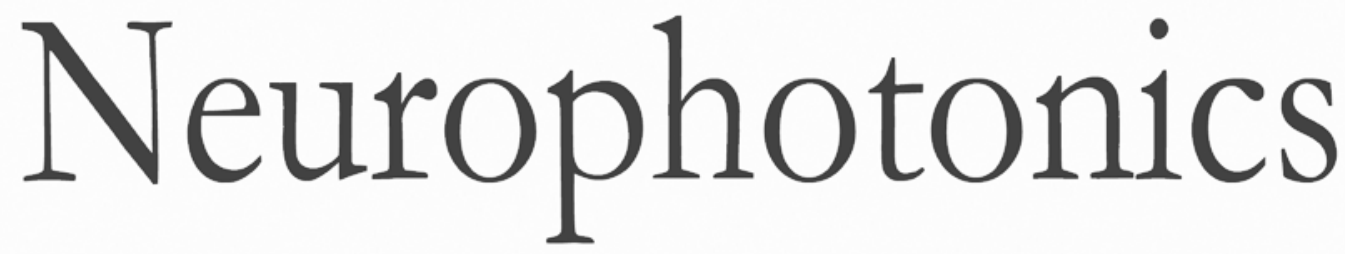

\title{
Mapping cortical network effects of fatigue during a handgrip task by functional near-infrared spectroscopy in physically active and inactive subjects
}

Elizabeth L. Urquhart

Hashini I. Wanniarachchi

Xinlong Wang

Hanli Liu

Paul J. Fadel

George Alexandrakis 


\title{
Mapping cortical network effects of fatigue during a handgrip task by functional near-infrared spectroscopy in physically active and inactive subjects
}

\author{
Elizabeth L. Urquhart, ${ }^{a}$ Hashini I. Wanniarachchi, ${ }^{a}$ Xinlong Wang, ${ }^{a}$ Hanli Liu, ${ }^{a}$ Paul J. Fadel, ${ }^{\text {b }}$ and \\ George Alexandrakis ${ }^{a, *}$ \\ aUniversity of Texas at Arlington, Bioengineering Department, Arlington, Texas, United States \\ bUniversity of Texas at Arlington, Department of Kinesiology, Arlington, Texas, United States
}

\begin{abstract}
The temporal evolution of cortical activation and connectivity patterns during a fatiguing handgrip task were studied by functional near-infrared spectroscopy (fNIRS). Twenty-three young adults (18 to 35 years old) were recruited to use a handheld force sensor to perform intermittent handgrip contractions with their dominant hand at their personal maximum voluntary contraction force level for $3.5 \mathrm{~s}$ followed by $6.5 \mathrm{~s}$ of rest for 120 blocks. Subjects were divided into self-reported physically active and inactive groups, and their hemodynamic activity over the prefrontal and sensory-motor cortices (111 channels) was mapped while they performed this task. Using this fNIRS setup, a more detailed time sequence of cortical activation and connectivity patterns was observed compared to prior studies. A temporal evolution sequence of hemodynamic activation patterns was noted, which was different between the active and the inactive groups. Physically active subjects demonstrated delayed fatigue onset and significantly longer-lasting and more spatially extended functional connectivity (FC) patterns, compared to inactive subjects. The observed differences in activation and FC suggested differences in cortical network adaptation patterns as fatigue set in, which were dependent on subjects' physical activity. The findings of this study suggest that physical activity increases FC with regions involved in motor task control and correlates to extended fatigue onset and enhanced performance. $\odot$ The Authors. Published by SPIE under a Creative Commons Attribution 4.0 Unported License. Distribution or reproduction of this work in whole or in part requires full attribution of the original publication, including its DOI. [DOI: 10 .1117/1.NPh.6.4.045011]
\end{abstract}

Keywords: functional near-infrared spectroscopy; cardiovascular health; handgrip exercise; fatigue; sensory-motor cortex; prefrontal cortex; functional connectivity.

Paper 19081RR received Aug. 20, 2019; accepted for publication Nov. 19, 2019; published online Dec. 10, 2019.

\section{Introduction}

When performing physical exercise, the contracting muscles elicit demand for oxygen, which is supplied by increased blood flow. The brain must register and simultaneously integrate input from feedforward (i.e., central command) and feedback (e.g., exercise pressor reflex) neural mechanisms to make necessary cardiovascular adjustments to meet the metabolic demand of the exercise. ${ }^{1,2}$ During exercise involving the arms, peripheral fatigue sets in, as characterized by reduced force-generating capability of the muscles that subsequently evolves into central fatigue, resulting in decreased neural drive to the muscles, after prolonged physical activity. ${ }^{1-6}$ Central fatigue's contribution to peripheral fatigue is less understood and functional brain imaging during fatiguing exercises is being investigated as a tool to help elucidate the underlying mechanisms. ${ }^{3-11}$ Neural pathways gradually alter their connectivity (neuroplasticity), which affects regulation of the cardiovascular system both at rest and during exercise. Physically active individuals exhibit exercise-related neuroplasticity and have improved cardiovascular health, whereas inactive individuals may be predisposed to higher incidences of cardiovascular disease. ${ }^{1,5,12}$

Several modalities have been used for functional brain mapping during motor-fatigue exercise, such as electroencephalography

*Address all correspondence to George Alexandrakis, E-mail: galex@uta.edu
(EEG), functional magnetic resonance image (fMRI), and functional near-infrared spectroscopy (fNIRS). ${ }^{3-8}$ Among them, fNIRS measures noninvasively the concentration changes of oxyhemoglobin $(\Delta \mathrm{HbO})$ and deoxyhemoglobin $(\Delta \mathrm{Hb})$ resulting from neurovascular coupling secondary to neuronal activation by utilizing near-infrared light (650 to $1000 \mathrm{~nm}$ wavelengths). fNIRS is advantageous because of its relatively lower cost, safety, portability, robustness to motion artifacts, and higher sensitivity compared to fMRI. ${ }^{13,14}$

While several functional neuroimaging studies have examined the effects of physical fatigue on brain activation and connectivity, ${ }^{3,4,6-11,15-18}$ none have explored how brain activity is modulated during peripheral fatigue in subjects of differing physical activity levels or shown the progressive effect of fatigue on brain activity. In healthy adult populations, fMRI has been used to show brain activation and connectivity continually during, ${ }^{17,18}$ or at the beginning and end of the exercise, ${ }^{6,7}$ to illustrate changing central motor command and strengthened functional connectivity (FC). In contrast, fNIRS optodes placed on the prefrontal cortex (PFC) ${ }^{9-11}$ and primary motor cortex $(\mathrm{M} 1)^{9}$ in trained athletic adult populations illustrated hyperoxygenation in the PFC and deoxygenation in M1 as a result of prolonged fatiguing exercise. However, those studies were unable to provide further insight on brain activity patterns due to the limited number of optodes used. Only one fNIRS study to date has, to our knowledge, examined the differences in brain 
activity between athletes and nonathletes during exhaustive sustained handgrip exercise but again only two probes were used, thus limiting whole-brain interpretations of activation and network connectivity. ${ }^{4}$ Therefore, prior functional neuroimaging studies have not provided a more global picture of the continuous temporal evolution of brain activity patterns and their differences based on subject physical activity levels during a fatiguing handgrip task.

To address this knowledge gap, the purpose of this study was to examine differences in cortical activity, as mapped by fNIRS, between physically inactive and active subjects during a maximal voluntary contraction (MVC) handgrip task. The temporal evolution of recorded hemodynamic activation and FC patterns was measured and compared. Analyzing brain network activation and connectivity allowed for detection of physical activitydependent network reorganization during a fatiguing motor task, which in the future could be explored as a novel means of evaluating exercise-induced functional changes in brain activation patterns in human health and disease.

\section{Materials and Methods}

\subsection{Participants}

Twenty-three young adults were recruited (four females, ages = $25.13 \pm 3.72$ years) for this study. All subjects were without any neurological or psychiatric disorders (self-reported). All but two subjects were right-handed, as determined by the Edinburgh handedness scale. ${ }^{19}$ Subjects also self-reported as physically inactive $(n=12$, exercising less than twice a week for $30 \mathrm{~min}$ of moderately vigorous exercise) or active ( $n=11$, exercising at least 4 times a week, for $30 \mathrm{~min}$ of moderately vigorous exercise). ${ }^{20}$ All experimental procedures, including a written consent required prior to participation in this study, were approved by the Institutional Review Board of the University of Texas at Arlington (IRB\# 2018-0686).

\subsection{Experimental Procedures}

An fNIRS imaging system (LABNIRS, Shimadzu Corp., Kyoto, Japan) was used to measure cerebral hemodynamic responses in the regions of interests (ROIs): left and right dorsolateral prefrontal cortex (IDLPFC; rDLPFC), left and right premotor cortex (IPMC; rPMC), left and right primary motor and sensory cortical (IM1/S1; rM1/S1) areas, and Broca's area. The optode layout on the subject's head consisted of 32 source fibers and 34 detector fibers that were arranged in a configuration resulting in a total of 111 channels with a source-detector distance of $3 \mathrm{~cm}$ for all that covered the aforementioned cortical areas, with no short-distance channel placement being available in that cap geometry [Fig. 1(a)]. Each source fiber was connected to the laser diodes at three wavelengths $(780,805$, and $830 \mathrm{~nm}$ ). The back-reflected light collected by the detector optodes was converted to current by photomultiplier tubes, and the resulting intensity data were sampled at a rate of $10.101 \mathrm{~Hz}$. The anatomical location of the optodes in relation to the standard head landmarks, including inion, nasion, $\mathrm{Cz}$, and left and right ears, were recorded for each subject using a three-dimensional digitizer (FASTRAK, Polhemus, Vermont). Montreal Neurological Institute (MNI) coordinates for the channels were determined using the statistical parametric mapping NIRS_SPM software package, which provided the Brodmann area (BA) corresponding to each fNIRS channel, as shown in Table S1 in the Supplementary Material. ${ }^{21}$

Subjects were seated at a table with their dominant upper arm at their side with their elbow flexed at 90 deg relative to the
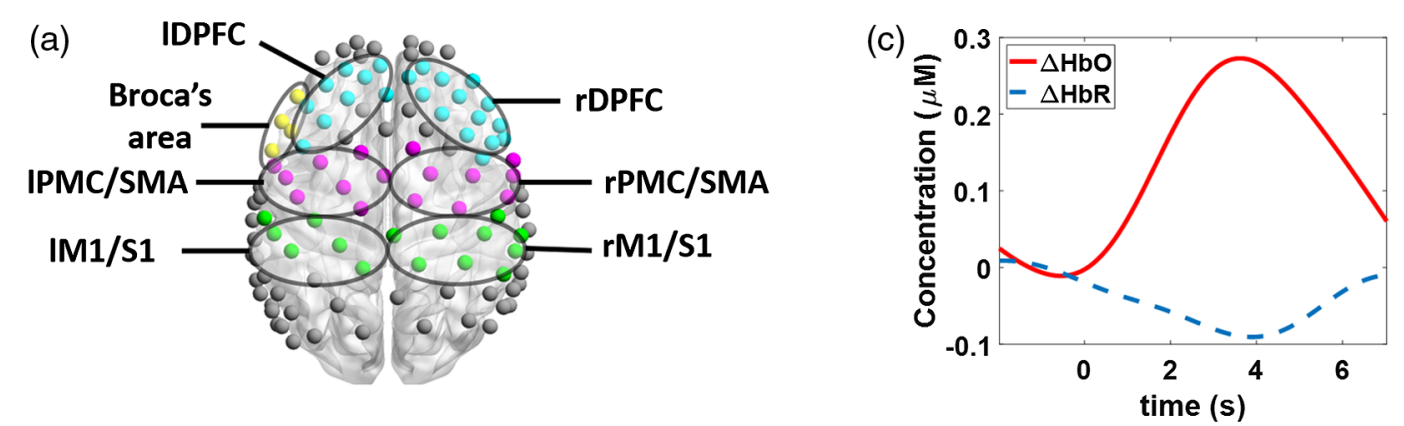

(b)

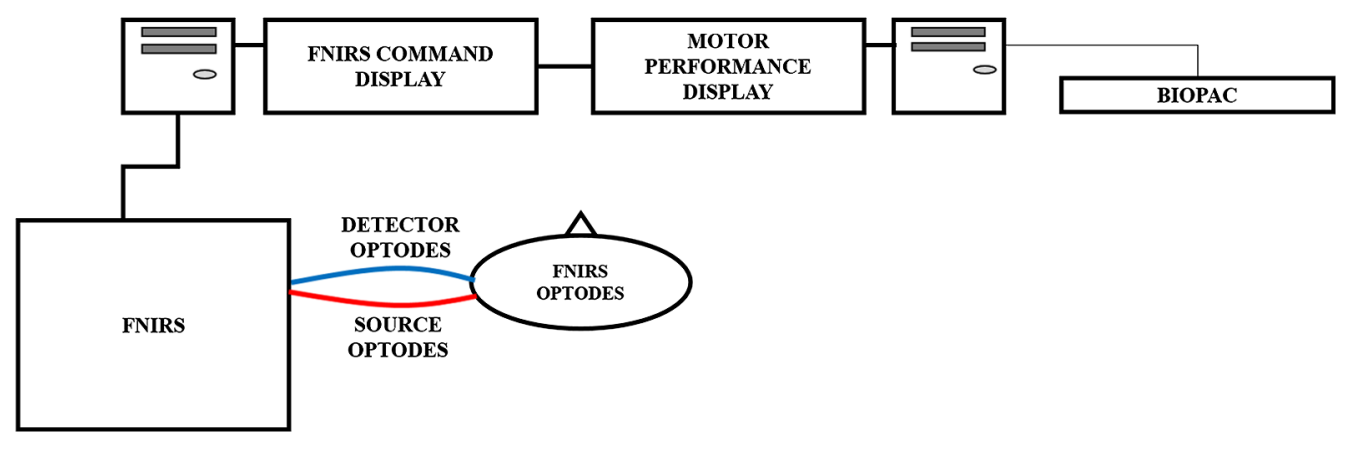

Fig. 1 Experimental setup and protocol timeline for the handgrip task. (a) The fNIRS multichannel layout with 111 channels covering five ROls: M1/S1 (green), PMC (red), DLPFC (blue), and Broca's area (yellow). All other channels, located over the temporal and occipital lobes are shown in gray. (b) Schematic representation of the experimental setup of the fNIRS system (LABNIRS) and the BIOPAC handgrip force sensor system with one representative source-detector channel shown for simplicity. (c) $\Delta \mathrm{HbO}$ and $\Delta \mathrm{HbR}$ hemodynamic responses at IM1/S1 for the first 10 blocks of the task. 
plane of the table on which their lower arm was supported and were facing two screens: one displaying protocol commands and the other displaying visual feedback of performance [Fig. 1(b)]. The fNIRS data acquisition began with a 5-min baseline hemodynamics measurement, followed immediately by imaging of the aforementioned cortical areas while subjects performed the handgrip task. For the latter, subjects used their dominant hand to perform intermittent handgrip contractions for $3.5 \mathrm{~s}$ alternating with $6.5 \mathrm{~s}$ of rest for 120 blocks at $100 \%$ MVC as a means to induce fatigue in the forearm. ${ }^{3,4,7,8}$ Prior to the experiment, subjects performed three to five MVCs and their average was calculated as the pretask MVC value for that subject. Handgrip force was measured by a hand dynamometer (BIOPAC, California) and displayed for visual feedback. Exerted force during the handgrip exercise was recorded using the hand dynamometer at $1-\mathrm{kHz}$ sampling rate. The maximum force value for each trial was calculated for each data point, and the resulting maximum force time-series data were low-pass filtered at $15 \mathrm{~Hz}^{8,22}$

\subsection{Mapping of Cerebral Hemodynamics}

FNIRS data were preprocessed using MATLAB 2012b (MathWorks, Natick, Massachusetts) and the open-source package Homer 2.0 [Fig. 1(c)]. ${ }^{14}$ Detrending was implemented using the least-squares fit of a line that was subtracted from the data. ${ }^{23}$ The raw intensity data were then low-pass filtered using a thirdorder Butterworth filter at a cut-off frequency of $0.2 \mathrm{~Hz}$ to remove large portions of physiological noise, including heartbeat $(1$ to $1.5 \mathrm{~Hz})$ and respiration $(0.2$ to $0.5 \mathrm{~Hz}){ }^{13}$ The fNIRS data were also high-pass filtered using a fifth-order Butterworth filter at a cut-off frequency of $0.01 \mathrm{~Hz}$ to remove any possible slow baseline drift. In addition, data from lefthanded subjects were flipped to its mirror image for group averaging purposes and the subsequent interpretation for all data was right (r) for contralateral and left (l) for ipsilateral brain hemispheres relative to the arm performing the task, as done in previous work. ${ }^{24}$ Optical density data were converted into changes in hemoglobin concentration relative to baseline $(\Delta \mathrm{HbO})$ using the modified Beer-Lambert law with an estimated differential pathlength factor of 6.0 for each wavelength, an estimate used in Homer 2.0. ${ }^{25}$ Lastly, a principal component analysis (PCA) filter was utilized to remove the first and second principal components, which are often associated with motion artifacts ${ }^{14}$ and global hemodynamic flucations, ${ }^{13}$ which may overlap with the task-related hemodynamic response frequencies.

General linear model (GLM) analysis was used to quantify time-dependent $\Delta \mathrm{HbO}$ patterns elicited during the handgrip task, by using a series of consecutive stimulation-specific boxcar functions convolved with a hemodynamic response function as a regressor, as in prior studies. ${ }^{26-28}$ Only $\Delta \mathrm{HbO}$ values were analyzed and reported in this study, because $\Delta \mathrm{HbR}$ values were found to have similar and opposite qualitative trends but with smaller amplitudes and lower signal-to-noise ratio, as previously reported in other studies for other motor activation tasks and as seen in Fig. 1(c). ${ }^{28,29}$ Subject group-level hemodynamic analyses were initially performed between baseline and during the task for each channel using a one-sample $t$-tests on $\beta$ values obtained from GLM, with multiple comparison corrections [Bonferroni and false discovery rate (FDR)]. Subsequently, channels belonging to the same ROI on a group level, as determined by NIRS_SPM, were averaged together and tested using FDR. ${ }^{30}$ Cortical activation images were visualized with the open-source network visualization tool BrainNet Viewer, ${ }^{31}$ using reference MNI coordinates that were not significantly $(p>0.05)$ different from the averaged MNI coordinates.

\subsection{Functional Connectivity Analysis}

FC was quantified using the open-source FC_NIRS software package. ${ }^{23}$ The 5-min baseline data were converted to resting state connectivity maps. Connectivity maps were created for two contiguous 10 -min periods: 0 to 10 and 10 to $20 \mathrm{~min}$ of the handgrip task. The raw optical density measurements were preprocessed the same way, as described in Sec. 2.3. Seed-based correlation analysis was performed by calculating the connectivity strength between the seed channel in cortical regions that showed significant activation during the task (rM1, IDLPFC, and rDLPFC) and every other channel via Pearson's correlation for every subject. ${ }^{23}$ The seed channel was chosen based on highest percentage overlap to the desired BA, as determined by NIRS_SPM. For FC analysis, one-sample $t$-tests were performed on the Pearson's correlation coefficient $(r)$ values of $\Delta \mathrm{HbO}$ across subjects at $p<0.05$ and were Bonferronicorrected for multiple comparisons of 111 channels. Topographical images for FC were generated using EasyTopo, an optical topography toolbox, which projects data on a standard brain MRI atlas and implements two-dimensional angular interpolation of the channel-wise data, for this study one-sample $t$-test $t$-values, in a spherical coordinate system. ${ }^{32}$

For statistical comparisons, $111 r$-values of $\Delta \mathrm{HbO}$ were averaged into one value, designated as $r_{a}$, for each subject for each period. A two-sample $t$-test was performed at $p<0.05$ on the averaged $r$-values between groups for each period. Paired $t$-tests were performed at $p<0.05$ on the averaged $r$-values to compare FC across time within each group.

Lastly, the linear relationship between regional FC magnitude and grip strength was examined using Pearson's correlation analysis. This was done for each ROI at each time point per subject group. To calculate differences between the $r$-values, the following equation was used: ${ }^{33}$

$$
z_{\text {observed }}=\frac{z_{1}-z_{2}}{\sqrt{\left(\frac{1}{N_{1}-3}\right)\left(\frac{1}{N_{2}-3}\right)}},
$$

where $z_{1}$ and $z_{2}$ are the Fisher's $r$-to- $z$ transformed values and $N_{1}$ and $N_{2}$ are the number of pairwise comparisons.

\section{Results}

\subsection{Evolution of Maximal Handgrip Force over Time}

The loss of handgrip force generated while intending to attain $100 \%$ of the MVC, recorded just prior to the beginning of the task, was quantified as a proxy measure of fatigue for inactive and active subjects. Force data were averaged over 60 blocks resulting in two time periods across the 120 contractions. The data blocks within each of the two time periods were tested for homogeneity of variances and normality, the assumptions for independent $t$-tests, which they did not pass. Therefore, data were analyzed using the nonparametric Mann-Whitney U test. ${ }^{34}$ The relative decrease in MVC force between active and inactive subjects was comparable, but the absolute force produced by the active subjects was consistently higher at each period: 0 to 10 and 10 to $20 \mathrm{~min}$ (Fig. 2; Table 1). 


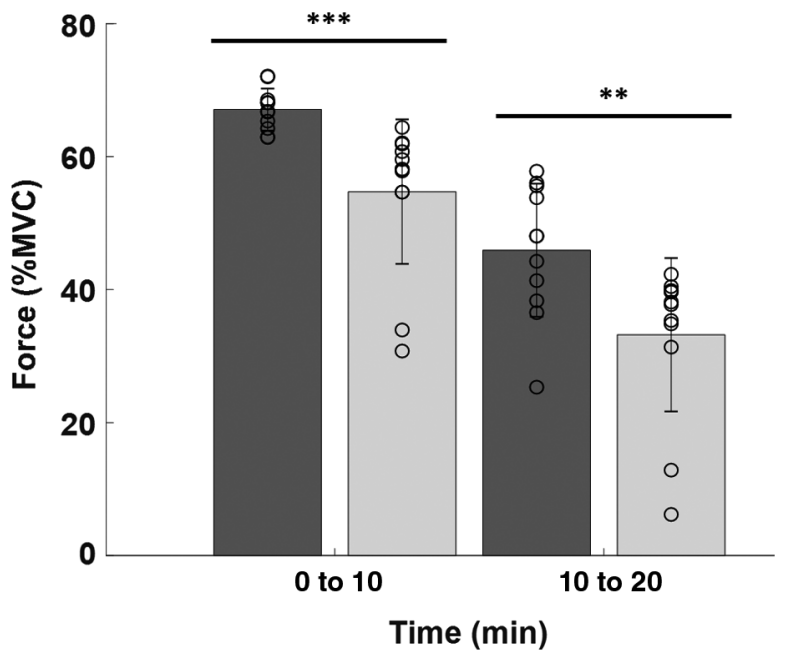

Fig. 2 Force produced during intermittent handgrip contractions at $100 \% \mathrm{MVC}$ force level using the dominant hand for physically inactive (light gray) and active subjects (dark gray). Each data point represents an average of 60 consecutive trials, expressed as the mean (bar height) \pm standard error to the mean (SEM; error bar). Circles: individual performance. ${ }^{*} p<0.01 ;{ }^{* *} p<0.001$.

Table 1 Mann-Whitney U statistics results of force (\%MVC) between active and inactive subjects.

\begin{tabular}{lcccc}
$\begin{array}{l}\text { Time period } \\
\text { (min) }\end{array}$ & $\begin{array}{c}\text { Mann-Whitney } \\
\text { U }\end{array}$ & z-statistic & $\begin{array}{c}\text { Effect } \\
\text { size }\end{array}$ & $p$-value \\
\hline 0 to 10 & 195 & 3.85 & 0.80 & 0.0001 \\
10 to 20 & 175 & 2.62 & 0.55 & 0.01 \\
\hline
\end{tabular}

\subsection{Temporal Evolution of Functional Near-Infrared Spectroscopy Activation Patterns}

While activation results were intended to be displayed in $\Delta \mathrm{HbO}$ activation maps, the high variability (Fig. S1 in the Supplementary Material) induced during the task did not allow for identification of statistically significant changes in individual channels when Bonferroni multiple comparisons correction was applied in this work. As a result, channels were grouped by ROI and averaged so that significance could be obtained at the expense of spatial localization (Fig. 3). Following this analysis approach, significant activation was found in Broca's area for inactive subjects within the first $100 \mathrm{~s}$ of the task, as indicated by red oval [Fig. 3(a)]. In contrast, active subjects showed significant activation in the IPMC and concurrent deactivation in the rDLPFC during the initial $100 \mathrm{~s}$ of the handgrip task [Fig. 3(d)]. However, there were no significant differences between inactive and active subjects at these early times [Fig. 3(g)]. In addition, while no statistical significance was found for any of the ROIs in the first half of the task [1 to $10 \mathrm{~min}$; Fig. 3(b)] due to high hemodynamic signal variability and low $\Delta \mathrm{HbO}$ amplitudes, in the second half of the task (11 to $20 \mathrm{~min}$ ) inactive subjects showed statistically significantly deactivation in the rDLPFC and rPMC, as indicated by the blue ovals in Fig. 3(c). Active subjects, on the other hand, while they also had high hemodynamic signal variability in the first half of the task, also had higher $\Delta \mathrm{HbO}$ magnitudes in certain cortical regions, which enabled detecting statistically significant activation in the 1M1/S1 and Broca's area [Fig. 3(e)]. Active subjects also showed significant deactivation in the rDLPFC during the first half of the task [Fig. 3(e)]. Interestingly, in the second half of the task, rDLPFC was not significant and instead activation was seen in the IDLPFC [Fig. 3(f)], opposite to what was seen for inactive subjects [Fig. 3(e)]. In addition, the rPMC became significantly deactivated in the second half of the task [Fig. 3(f)], similar to what was observed for the inactive subjects [Fig. 3(e)]. Lastly, active subjects were significantly activated in Broca's area [Fig. 3(h)] in the first half of the task and in IDLPFC [Fig. 3(i)] in the second half of the task, compared to inactive subjects.

\subsection{Temporal Evolution of Functional Connectivity Patterns}

\subsubsection{Functional connectivity maps with seed at left primary motor cortical area}

The IM1 seed location was placed based on the activation seen in Fig. 3(e) and by channel location as determined by NIRS SPM. The FC maps at rM1, contralateral to the hand performing the handgrip task, are shown in Fig. 4 for inactive (upper row) and active subjects (lower row). During the handgrip task, inactive subjects initially exhibited statistically significant FC between the 1M1 and the dorsolateral prefrontal cortex (DLPFC) and rPMC [Fig. 4(b)]. However, as the task progressed, FC patterns became more localized to the $1 \mathrm{M} 1$ with simultaneous loss of connectivity strength with the IDLPFC and receding connectivity strength in the rM1/S1 and the rDLPFC [Fig. 4(c)]. Active subjects, on the other hand, showed spatially broader FC patterns when performing the same task. In particular, active subjects exhibited significant FC with the DLPFC, the rM1/S1, and the rPMC throughout the entire duration of the task [Figs. 4(e) and 4(f)]. The $r_{a}$ values were significantly greater in active subjects than inactive subjects at each time period: baseline ( $p=$ $0.03), 0$ to $10(p=0.02)$, and 10 to $20 \mathrm{~min}(p=0.02)$. As well, $r_{a}$ values within groups were significantly different from one another across time for inactive subjects at baseline and 0 to $10 \min (p=0.003)$ and 0 to 10 and 10 to $20 \min (i=0.02)$ and active subjects at baseline and 0 to $10 \mathrm{~min}(p=0.05)$ and 0 to 10 and 10 to $20 \mathrm{~min}(p=0.01)$.

\subsubsection{Functional connectivity maps with seed at left dorso- lateral prefrontal cortex}

When placing the seed at IDLPFC, the generated FC maps also showed major differences between the inactive and the active subject groups (Fig. 5). Inactive and active subjects within the first $10 \mathrm{~min}$ of the task displayed statistically significant FC strength between the IDLPFC and the rDLPFC, PMC, and M1/S1 [Figs. 5(b) and 5(e)]. However, in the second half of the task, the inactive subjects' FC pattern receded toward the DLPFC [Fig. 5(c)], whereas the active subjects' FC pattern broadened to the IPMC and somatosensory association cortex [Fig. 5(f)]. The $r_{a}$ values were significantly greater in active subjects than inactive subjects at 0 to $10(p=0.01)$ and 10 to $20 \min (p=0.02)$ but not at baseline $(p=0.06)$. The $r_{a}$ values within groups were only significantly different across time at baseline and at 0 to $10 \mathrm{~min}$ for inactive $(p=0.002)$ and active subjects $(p=0.01)$. 

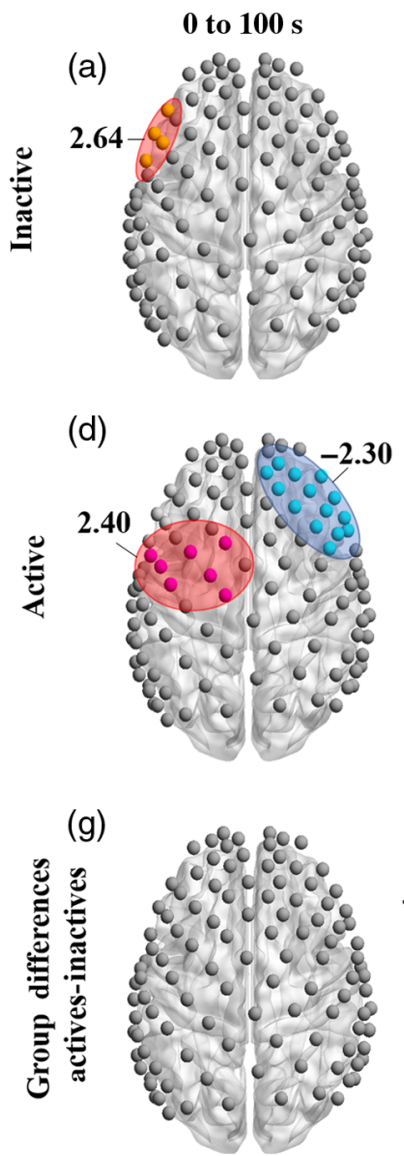

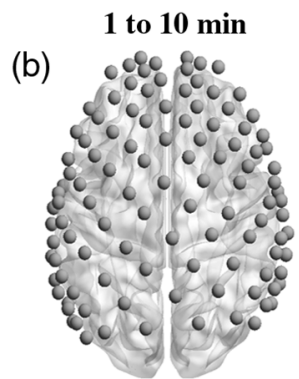

(e)

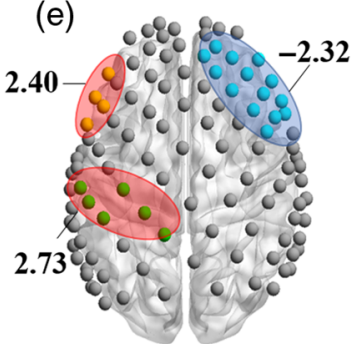

(h)

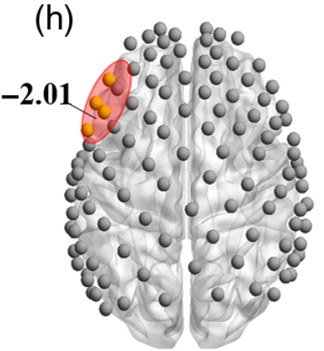

11 to $20 \mathrm{~min}$

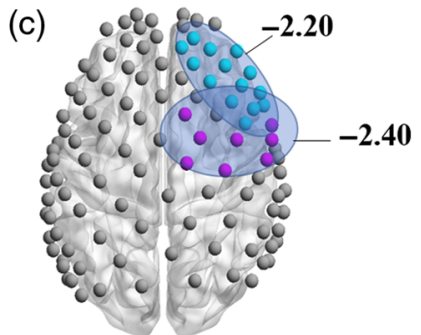

(f)
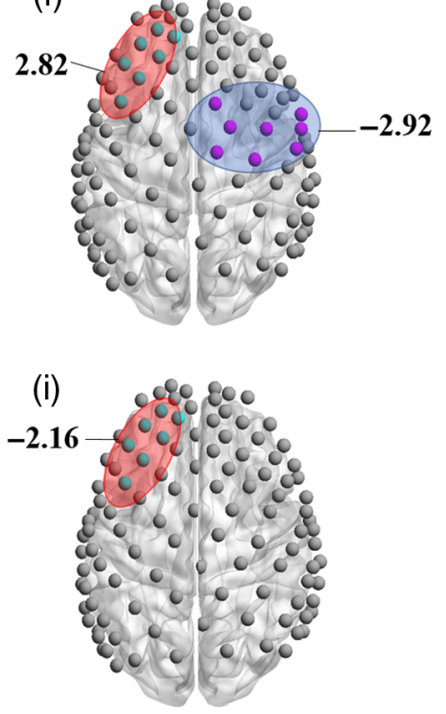

Fig. 3 ROIs of statistically significant activity for inactive subject's at (a) 0 to $100 \mathrm{~s}$, (b) 1 to $10 \mathrm{~min}$, and (c) 11 to $20 \mathrm{~min}$ and for active subjects at (d) 0 to $100 \mathrm{~s}$, (e) 1 to $10 \mathrm{~min}$, and (f) 10 to $20 \mathrm{~min}$ of the handgrip task. Only ROls with statistically significant $(p<0.05$, FDR corrected) activation are shown with corresponding $t$-values next to it (red ovals-activation; blue ovals-deactivation). No significant group differences seen early in the handgrip task at (g) 0 to $100 \mathrm{~s}$, and greater activation in few regions was seen for active subjects (red ovals; negative $t$-values) over longer time intervals; (h) 0 to $10 \mathrm{~min}$, and (i) 10 to $20 \mathrm{~min}$. With the exception of (h) and (i), all positive $t$-values corresponded to activation (red ovals) and all negative $t$-values corresponded to deactivation (blue ovals).
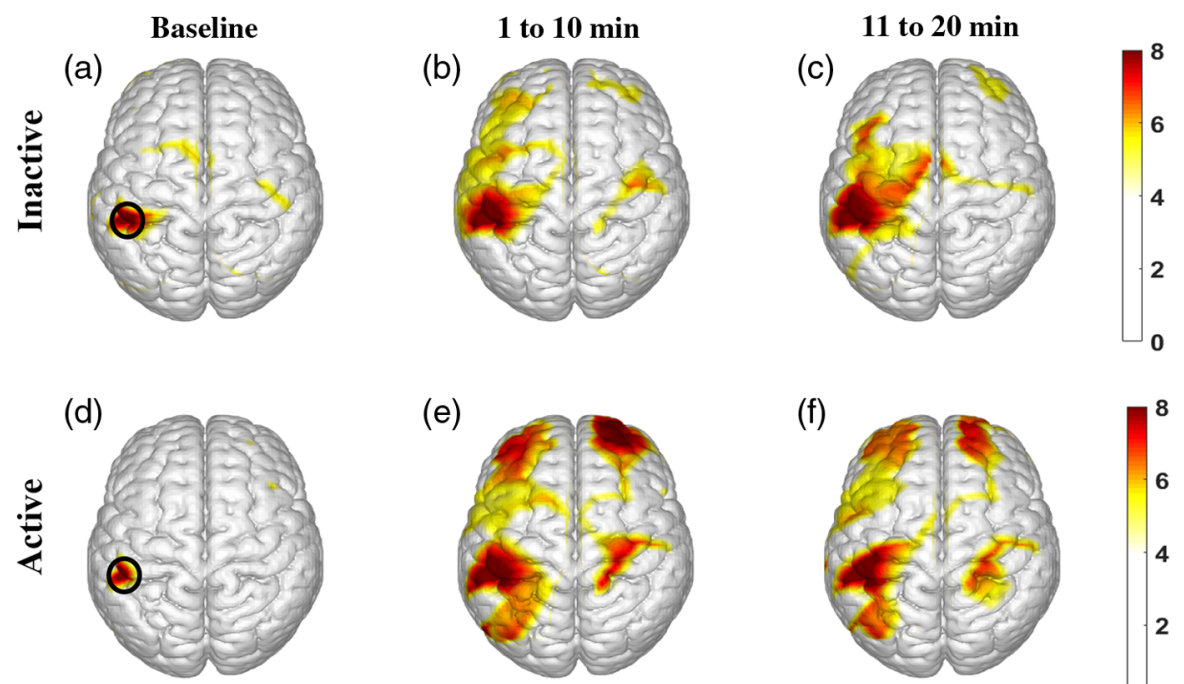

(e)

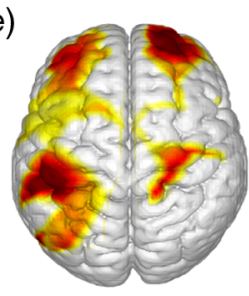

(f)

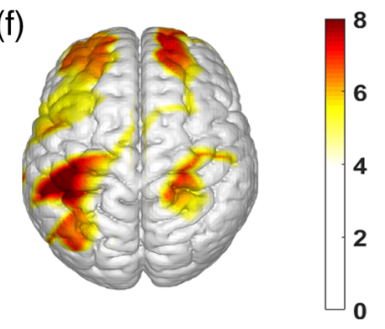

$t$-score

Fig. 4 Evolution of FC patterns during the entire handgrip task with the seed region at IM1 for inactive subjects at (a) baseline, (b) 0 to $10 \mathrm{~min}$, and (c) 10 to $20 \mathrm{~min}$ and for active subjects at (d) baseline, (e) 0 to $10 \mathrm{~min}$, and (f) 10 to $20 \mathrm{~min}$. The black oval encircles the seed region channels and is only displayed at baseline for clarity. Only regions with statistically significant FC strength are shown $(p<0.05$, Bonferroni-corrected). 

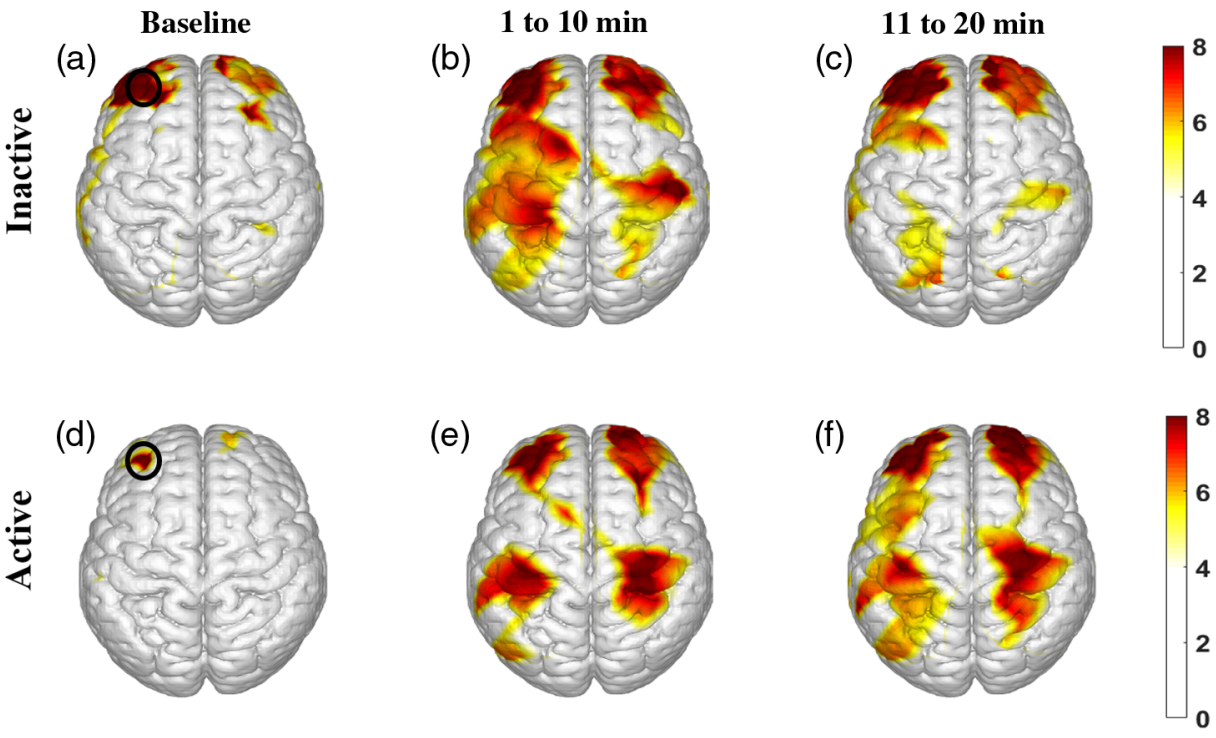

$t$-score

Fig. 5 Evolution of FC patterns during the entire handgrip task with the seed region at IDLPFC for inactive subjects at (a) baseline, (b) 0 to $10 \mathrm{~min}$, and (c) 10 to $20 \mathrm{~min}$, and for active subjects at (d) baseline, (e) 0 to $10 \mathrm{~min}$, and (f) 10 to $20 \mathrm{~min}$. The black oval encircles the seed region channels and is only displayed at baseline for clarity. Only regions with statistically significant FC strength are shown $(p<0.05$, Bonferroni-corrected).

\subsubsection{Functional connectivity maps with seed at right dorsolateral prefrontal cortex}

The FC maps with the seed at rDLPFC, the symmetrically contralateral position to the IDLPFC seed considered above, are shown in Fig. 6. Within the first $10 \mathrm{~min}$ of the task, inactive subjects exhibited statistically significant but relatively weak FC strength to the IDLPFC, rPMC, and IM1/S1 [Fig. 6(b)]. In the subsequent 10 min of the task, there was loss of FC strength with the rPMC [Fig. 6(c)]. In contrast, active subjects displayed significant FC strength with the IDLPFC, bilateral M1/S1, and some weaker but significant FC to bilateral PMC within the first 10 min [Fig. 6(e)], which weakened in the second $10 \mathrm{~min}$ of the task [Fig. 6(f)]. The $r_{a}$ values were significantly greater in active subjects than in inactive subjects at each time period: baseline $(p=0.02), 0$ to $10(p=0.01)$, and 10 to $20 \mathrm{~min}$ $(p=0.04)$. The $r_{a}$ values within groups were also significantly different from one another across time for inactive subjects at
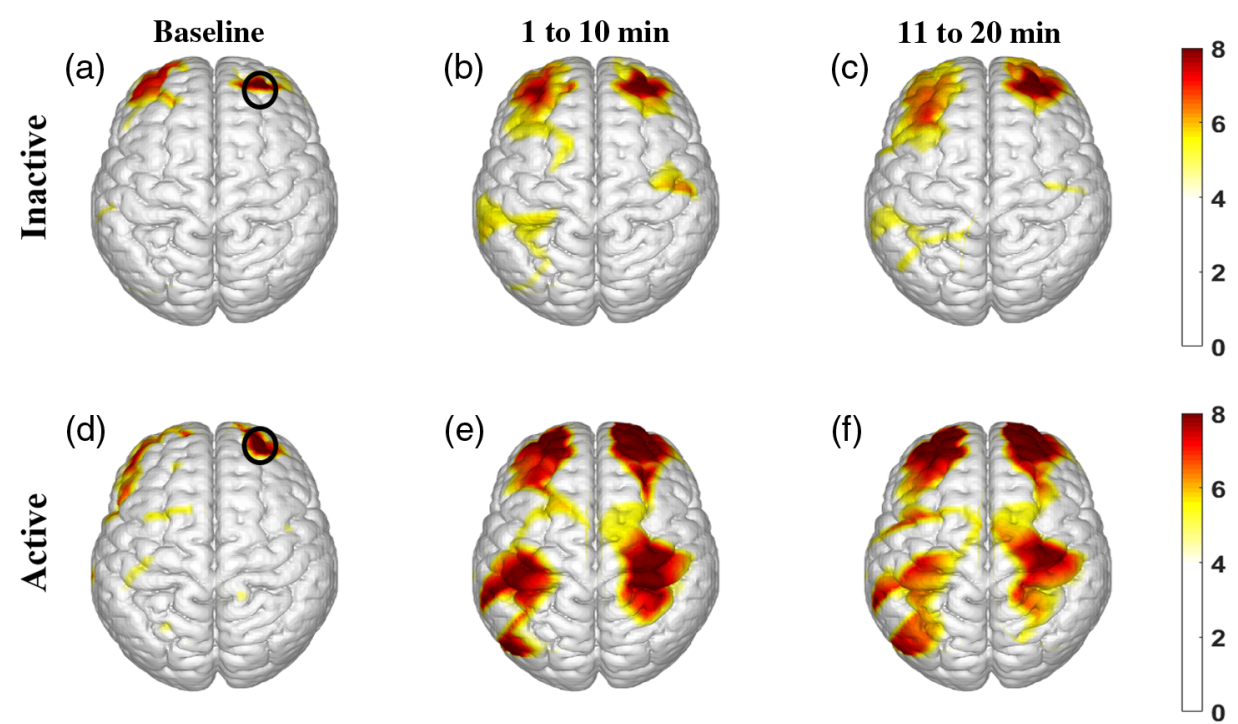

$t$-score

Fig. 6 Evolution of FC patterns during the entire handgrip task with the seed region at rDLPFC for inactive subjects at (a) baseline, (b) 0 to $10 \mathrm{~min}$, and (c) 10 to $20 \mathrm{~min}$, and for active subjects at (d) baseline, (e) 0 to $10 \mathrm{~min}$, and (f) 10 to $20 \mathrm{~min}$. The black oval encircles the seed region channels and is only displayed at baseline for clarity. Only regions with statistically significant FC strength are shown $(p<0.05$, Bonferroni-corrected). 
Table 2 Pearson's correlation analysis summary between regional FC magnitude and time to $50 \%$ MVC [Pearson's correlation coefficient (r) and $p$-value].

\begin{tabular}{|c|c|c|c|c|c|c|c|}
\hline & & \multicolumn{2}{|c|}{ IM1 } & \multicolumn{2}{|c|}{ IDLPFC } & \multicolumn{2}{|c|}{ rDLPFC } \\
\hline & & $r$ & $\mathrm{p}$ & $r$ & $\mathrm{p}$ & $r$ & $\mathrm{p}$ \\
\hline \multirow[t]{3}{*}{ Inactive } & Baseline & -0.35 & 0.26 & -0.56 & 0.06 & -0.17 & 0.59 \\
\hline & 0 to $10 \mathrm{~min}$ & -0.17 & 0.59 & -0.5 & 0.10 & -0.37 & 0.24 \\
\hline & 10 to $20 \mathrm{~min}$ & -0.31 & 0.33 & -0.45 & 0.15 & -0.45 & 0.15 \\
\hline \multirow[t]{3}{*}{ Active } & Baseline & 0.63 & 0.04 & 0.74 & 0.01 & 0.69 & 0.02 \\
\hline & 0 to $10 \mathrm{~min}$ & 0.69 & 0.02 & 0.65 & 0.03 & 0.49 & 0.13 \\
\hline & 10 to $20 \mathrm{~min}$ & 0.63 & 0.04 & 0.59 & 0.05 & 0.43 & 0.13 \\
\hline
\end{tabular}

Table 3 Correlation coefficients comparison summary between inactive and active subjects for regional FC magnitude and 50\% MVC ( $z$-value and $p$-value).

\begin{tabular}{|c|c|c|c|c|c|c|}
\hline & \multicolumn{2}{|c|}{ IM1 } & \multicolumn{2}{|c|}{ IDLPFC } & \multicolumn{2}{|c|}{ rDLPFC } \\
\hline & $z$ & $\mathrm{p}$ & z & $\mathrm{p}$ & $z$ & $p$ \\
\hline Baseline & -2.28 & 0.02 & -3.26 & 0.001 & -2.1 & 0.04 \\
\hline 0 to $10 \mathrm{~min}$ & -1.88 & 0.06 & -2.73 & 0.006 & -1.9 & 0.06 \\
\hline 10 to $20 \mathrm{~min}$ & -2.19 & 0.03 & -2.39 & 0.02 & -1.94 & 0.05 \\
\hline
\end{tabular}

baseline and 0 to $10 \min (\mathrm{p}=0.003)$ only and active subjects at baseline and 0 to $10 \mathrm{~min}(p=0.03)$ and 0 to 10 and 10 to $20 \mathrm{~min}$ $(p=0.01)$.

\subsubsection{Correlation between regional functional connectivity and grip strength}

The $r$-values were quantified between regional FC magnitude and time to $50 \% \mathrm{MVC}$. This was performed for each group at each time point. In addition, correlation coefficients were compared between active and inactive subjects at each time point. Active subjects had significant $(p<0.05)$, positive correlation between 1M1, 1DLPFC, and rDLPFC and 50\%MVC at all time points, with the exception of rDLPFC at 0 to 10 and 10 to 20 min, whereas inactive subjects had no significant correlation (Table 2). Active subjects' correlation was more significant than inactive subjects' at $1 \mathrm{M} 1$ (baseline and 10 to $20 \mathrm{~min}$ ), IDLPFC (all time periods), and at rDLPFC (baseline and 10 to $20 \mathrm{~min}$ ) (Table 3).

\section{Discussion}

The present study aimed to map by fNIRS the temporal evolution in hemodynamic activation and FC patterns in physically inactive and active subjects performing a fatiguing handgrip task. The observed differences in cortical activity patterns and concurrently acquired handgrip force data suggest physical activity-dependent network reorganization across multiple cortical regions during this task.

\subsection{Changes in Handgrip Performance during the Task}

Physical fatigue induced by intermittent muscle contractions and its effect on force has been studied extensively in physiology $^{35,36}$ and recent neuroimaging studies. ${ }^{3,6,8,17,22,37,38}$ Fatigue has been defined as "any decline in muscle performance associated with muscle activity at the original intensity." 36 Both inactive and active subjects' muscle performance adhered to this definition vis-à-vis the gradual decline in MVC throughout the exercise. Moreover, our results are in agreement with a prior handgrip fatigue study showing that the rates of fatigue were similar between subjects with varying physical activity levels and initial strength, final strength, and absolute endurance were larger for the active subjects. ${ }^{35}$

\subsection{Evolution of Hemodynamic Activation Patterns during the Handgrip Task}

Previous functional neuroimaging studies concerning arm and hand movements have demonstrated activation in M1, PMC/ SMA, and PFC. ${ }^{6,15,17,37,39,40}$ In this work, inactive and active subjects exhibit significant activity in these regions and in Broca's area as well. However, as the task progressed, there were subsequent shifts in activity toward the DLPFC. In a prior fMRI study involving a handgrip task performed under nonfatiguing conditions (30\% MVC), as verified by electromyography (EMG), increased activation was seen bilaterally in M1/ S1 with concurrent EMG signal increased for several forearm muscles. ${ }^{17}$ In contrast, during a fatiguing handgrip task $(100 \%$ MVC), initial M1/S1 activation and concurrently acquired EMG signals declined, but supplementary cortical regions such as the PFC maintained consistent activation. ${ }^{18}$ While EMG measurements were not performed in this work, the above findings do support the notion that a stronger central command, via increased brain activation in the PFC, is needed to maintain task performance once fatigue sets in. However, prior studies do not discuss the temporal evolution of cortical activation and connectivity patterns during the handgrip task. These are discussed here in more detail with respect to the cortical areas involved.

\subsubsection{Evolution of hemodynamic activation patterns at primary and secondary sensory-motor cortices}

The primary sensory-motor cortex, or M1/S1, is responsible for motor control and execution ${ }^{7,41}$ and works in conjunction with the secondary sensory-motor cortex, or PMC, which is associated with movement planning and preparation. ${ }^{41}$ Physically active individuals were able to elicit brain activation and deactivation in these regions, similar to a prior neuroimaging motor task study. ${ }^{18}$ This work, however, differs from another prior study comparing athletes to nonathletes performing a sustained handgrip task, where athletes exhibited decreased M1 activation compared to nonathletes during a sustained handgrip task at $50 \%$ MVC. $^{4}$ As that study used only two fNIRS channels, it is possible that its results were affected by incomplete spatial coverage of the sensory-motor cortices, as the authors also suggested. ${ }^{4}$ Also, in this work, both athletes and nonathletes exhibited significant deactivation in the rPMC toward the end of the task, when subject fatigue was the highest. ${ }^{4}$ We hypothesize that an increase in variability of activation as a result of exercise hindered determination of activation significance in inactive subjects (see Fig. S1 in the Supplementary Material), 
as also demonstrated in a prior fMRI fatiguing handgrip study. ${ }^{6}$ That study noted a positive correlation between exercise duration and increased hemodynamic signal variance, with high variance correlating to low pixel activation. ${ }^{6}$

This work demonstrates substantial location shifts of focal regions during the fatiguing handgrip task from contralateral to ipsilateral regions and from posterior to anterior regions of higher brain activity, in agreement with an EEG fatiguing handgrip study. ${ }^{3}$ Moreover, brain activation changes in deeper subcortical structures participating in motor regulation (i.e., bilateral basal ganglia, cerebellum, and thalamus) are known to occur as a coordinated effort to optimize motor unit recruitment and activation level for prolonged fatiguing exercise. ${ }^{38,42}$ Although it would be reasonable to expect that these structures could also be activated as the handgrip task progressed, those regions were not accessible by fNIRS.

\subsubsection{Evolution of hemodynamic activation patterns in the dorsolateral prefrontal cortex}

In the context of motor tasks, the DLPFC is associated with motor preparation and planning over long periods of time, action selection, and control ${ }^{41}$ and correlates with higher force output. ${ }^{42,43}$ In a prior study, the DLPFC was activated predominantly on the side contralateral to the used hand, ${ }^{42}$ which is consistent with the increased IDLPFC activation observed in physically active subjects in our work. Other fNIRS studies that measured PFC activation during near-maximal or exhaustive aerobic exercise found that, as the near exhaustion was reached, DLPFC activation increased bilaterally. ${ }^{10,11}$ However, in these latter studies, subjects were trained athletes performing a bilateral task such as cycling and not a unilateral task, like in our work.

The rDLPFC is associated with inhibition or avoidance behavior toward meeting a goal and has been suggested to be involved during prolonged exercise to purposefully inhibit bodily afferences that arise with physical fatigue and preserved mental effort during exercise maintenance. ${ }^{11}$ In agreement with prior studies, the rDLPFC deactivation seen in our results correlated with a reduction in handgrip force. ${ }^{9-11,44}$ These prior studies suggest that subject stress due to the prolonged and physically challenging motor task may have contributed to the observed rDLPFC deactivation. The IDLPFC is associated with an approach reaction toward a goal and may suggest the willingness of the active subjects to challenge themselves and meet the task goal even in the presence of fatigue. ${ }^{44}$ We hypothesize that as the task progressed, active subjects adopted a goaloriented approach resulting in the dominance of IDLPFC activation, whereas inactive subjects adopted a goal-avoidance approach that resulted in the dominance of rDLPFC deactivation.

\subsubsection{Evolution of hemodynamic activation patterns at the Broca's area}

Inner speech refers to the activity of silent expression of conscious thought to oneself and results in activation in Broca's area in the left hemisphere. ${ }^{45}$ Sports literature has further studied inner speech of positive, negative, motivational, and instructional context known as self-talk via questionnaires in athletes, which found that athletes participate in self-talk more frequently in competition settings and when performing individually, as opposed to on a team. ${ }^{46-48}$ While the active subjects were not athletes, they too had significantly more activation in Broca's area than inactive subjects.

\subsection{Evolution of Functional Connectivity Patterns during the Handgrip Task}

The application of FC analysis to the fNIRS data collected in this study provided details of cortical area interconnectedness during the entire duration of the handgrip task than seen in prior neuroimaging studies using similar protocols, which only reported results for the beginning and the end of the task period. ${ }^{7,8,22,38}$ In our work, seed regions were specifically placed at locations with significant hemodynamic activity namely, the IM1, the IDLPFC, and the rDLPFC. These seed regions are all known to be connected to the descending motor pathways that control hand and arm movements. . $^{7-11,49}$

\subsubsection{Overall functional connectivity pattern differences between inactive and active subjects}

This study showed significant differences in FC patterns between inactive and active subjects when performing a fatiguing handgrip task. Active subjects exhibited more spatially extended FC patterns that persisted into the second half of the task, compared to inactive subjects who showed progressively diminished connectivity to areas distant to the seed region. Physical exercise is known to increase the brain function throughout life and has been shown to enhance FC in the default mode network, frontoparietal network, and motor network, as well as increase gray brain volume in the prefrontal, and temporal cortex, and the hippocampus. ${ }^{50-53}$ Physically active subjects' expansive connectivity patterns highlight the greater availability of cortical network resources due to prior exercise. Overall, the findings of our work are consistent with the existing notion of exercise-related augmentation in $\mathrm{FC}$ at the resting state and during fatiguing tasks. ${ }^{8}$

\subsubsection{Functional connectivity pattern comparisons with seed at left primary motor cortical area}

The seed at $1 \mathrm{M} 1$ indicated strengthened FC between bilateral DLPFC, bilateral PMC, and rM1/S1. However, the spatial extent, temporal persistence, and hemispheric localization were different between inactive and active subjects. The M1 region is the primary neural output center of the brain to the working muscles because of its vital role in motor control and execution during exercise. ${ }^{7,41}$ FC strength between $1 \mathrm{M} 1$ and bilateral DLPFC, which is associated with executive behavior control ${ }^{44,54}$ and involved in motor planning and preparation, ${ }^{9-11}$ was present throughout the task in both groups. However, only active subjects' FC strength toward bilateral DLPFC was consistent during the entire task, which is attributed as reinforcement of the top-down regulation to the primary and secondary motor cortices under fatiguing conditions. ${ }^{7,9}$ A prior neuroimaging study also reported enhanced connectivity in young adult endurance athletes compared to heathy controls, similar to the difference in FC strength to bilateral DLPFC seen between inactive and active subjects in this study. ${ }^{53}$ The FC patterns between IM1 and $\mathrm{rPMC}$ and $\mathrm{rM} 1 / \mathrm{S} 1$ regions illustrated the enhanced recruitment of cortical regions involved in motor planning and execution for active subjects compared to inactive subjects. Lastly, in prior fMRI work involving a similar protocol, the $1 \mathrm{M} 1$ connectivity to bilateral $\mathrm{S} 1$ in active subjects was suggested to be due to 
increased sensory feedback from the arm muscles to the central motor command. ${ }^{7}$

\subsubsection{Functional connectivity pattern comparisons with seeds at left dorsolateral prefrontal cortex and right dorsolateral prefrontal cortex}

The DLPFC is extensively connected with the sensory-motor cortex and is associated with regulating attention, goal-directed behavior, thought, and motor planning and preparation. ${ }^{9-11,44,49,55}$ However, the IDLPFC and rDLPFC are further involved in the role of approach and avoidance behaviors, respectively, which engender differences in their connectivity with other cortical regions. ${ }^{11,44,55}$ As a result, our data not only showed that FC patterns had significant differences between active and inactive subjects for both seed locations, but it also showed slightly different FC patterns within each subject category, depending on whether the seed location was at the IDLPFC or the rDLPFC.

The IDLPFC is associated with the pursuit of approachrelated goals, ${ }^{56}$ such as maintaining MVC. The IDLPFC has also shown high connectivity strength with cortical regions that associated with the pursuit of approach-related goals, ${ }^{56}$ such as maintaining MVC. As the task progressed and handgrip force production decreased, the FC strength of the IDLPFC regions to other cortical areas diminished in inactive subjects but became more spatially extended in active subjects. Under fatigue conditions, noradrenaline and dopamine are released, which impair the top-down or executive control of the DLPFC and strengthen the bottom-up control, driven by the salience of the stimulus, through the amygdala, which result in a more reflexive and habitual motor responses. ${ }^{49}$ Prior FC studies have indicated that subcortical structures such as the amygdala, basal ganglia, and anterior cingulate cortex, not accessible by fNIRS, reinforce the descending command under fatigue conditions, which would contribute to maintaining task performance and possibly goal pursuit. ${ }^{7,44,55,56}$ Lastly, FC between the IDLPFC and other motor planning and control cortical regions is also dependent on subject motivation, which suggests that active subjects are more motivated than inactive subjects during this task. $^{56}$

The rDLPFC seed juxtaposes the difference in FC patterns between inactive and active subjects at each time period more clearly than the IDLPFC. This may be a result of the rDLPFC's specialized involvement in the maintenance of prolonged physical exercise due to its role in avoidance, or more specifically inhibition of impulse responses. ${ }^{11}$ We propose that active subjects have stronger, persistent FC between rDLPFC and IDLPFC, bilateral PMC, and bilateral M1/S1 because they were able to inhibit bodily afferences that arise with fatigue more successfully than their inactive counterparts. ${ }^{11}$ In addition, the rDLPFC strongest connection was to IDLPFC (Table S2 in the Supplementary Material), especially in active subjects at baseline (Table S3 in the Supplementary Material), possibly because inhibition was needed to maintain task performance that required stronger, longer-lasting FC to other higher-order DLPFC areas, as suggested in a prior functional neuroimaging study. ${ }^{9-11}$

\subsubsection{Correlation between functional connectivity and performance}

Correlation between regional FC and performance (i.e., time to $50 \%$ MVC) further exemplified the difference in FC strength between inactive and active subjects. Exercise has been suggested to improve neuronal activity and promote angiogenesis and vascular function to cortical brain regions, including the motor cortex. ${ }^{57}$ In addition, resistance exercise increases torque- and power-generating capacity in the muscle and also positively impacts functional plasticity in older subjects. ${ }^{58}$ This study further demonstrated that active subjects had a positive relationship between performance and FC strength in $1 \mathrm{M} 1$, IDLPFC, and rDLPFC at nearly every time point. In contrast, inactive subjects had no significant correlation between performance and FC strength possibly due to limited exercise-related structural and functional changes.

\section{Study Limitations}

This study has some limitations that should be considered. First, individuals were grouped based on a self-reported physical activity questionnaire that neglected to specify type of exercise (i.e., endurance or resistance) performed by the subject. This information could have better defined the active populations. In addition, fNIRS is limited in only measuring cortical brain areas and has lower spatial resolution compared to fMRI. ${ }^{13,14}$ Also, short-distance channels $(<1 \mathrm{~cm})$ were not employed in this study as they were not available in the commercial optode holder cap used. Signals measured by short-distance channels are dominated by systemic interferences from superficial scalp layers such as cardiac activity and respiration and can be regressed out. ${ }^{59}$ Short-distance channels could also provide blood flow changes in the extracerebral layer of the head due to the task. ${ }^{60}$ In addition, it is unknown which other method(s) would be the best approach in removing physiological noise. There exist several different ways to remove global interference due to the scalp and skull hemodynamics in addition to the PCA in our work. These methods include (1) short-distance channels, as previously mentioned; (2) use of ICA; (3) adaptive filtering; (4) calculating the mean signal over all channels and using the mean as a superficial regressor; or (5) a combination of these methods can be used for removal. ${ }^{14,61,62}$ Thus, a quantitative comparison using different methods is warranted in future studies.

\section{Conclusion}

This is the first study, to our knowledge, that presents a direct comparison of differences in the temporal evolution of cortical hemodynamic activation and FC patterns between physically active and inactive subjects during a fatiguing handgrip task. The observed patterns suggest that physical activity modifies both baseline connectivity and the way that different cortical regions are recruited as subjects try to maintain MVC for a prolonged time. Overall, hemodynamic activity moved from the sensory-motor areas early into the task toward the PMC and DLPFC as the task progressed. However, the temporal evolution of activation patterns was different between active subjects (30 min of moderately vigorous exercise at least 4 times a week) and inactive subjects (exercised less than twice a week), consistent with approach (active) versus avoidance (inactive) tendencies toward the task goal. At the same time, active subjects exhibited longer-lasting and broader connectivity patterns, which likely contributed to the sustenance of higher handgrip force output, compared to inactive subjects, as fatigue set in. These results provide preliminary evidence for broad network pattern differences across multiple cortical regions during a fatiguing task that are specific to subjects' physical activity. 
We propose to use this protocol in future work as a novel means of evaluating exercise-induced functional changes in brain activation patterns in human health and disease.

\section{Disclosures}

All of the authors had neither relevant financial or competing interests nor other potential conflicts of interest.

\section{Acknowledgments}

The authors acknowledge the financial support from the National Heart, Lung, and Blood Institute with the award number NIH T32 HL134613 for Elizabeth Urquhart (née Lyde). The content is solely the responsibility of the authors and does not necessarily represent the official views of the National Institutes of Health.

\section{References}

1. P. J. Mueller et al., "Integration of central and peripheral regulation of the circulation during exercise: acute and chronic adaptations," Compr. Physiol. 8(1), 103-151 (2017).

2. J. Gonzalez-Alonso et al., "Brain and central haemodynamics and oxygenation during maximal exercise in humans," J. Physiol. 557(1), 331-342 (2004).

3. J. Z. Liu et al., "Shifting of activation center in the brain during muscle fatigue: an explanation of minimal central fatigue?" Neuroimage 35(1), 299-307 (2007).

4. K. Shibuya and N. Kuboyama, "Decreased activation in the primary motor cortex area during middle-intensity hand grip exercise to exhaustion in athlete and nonathlete participants," Percept. Mot. Skills 111(1), 19-30 (2010).

5. S. W. Wong et al., "Sex differences in forebrain and cardiovagal responses at the onset of isometric handgrip exercise: a retrospective fMRI study," J. Appl. Physiol. 103(4), 1402-1411 (2007).

6. N. M. Benwell et al., "Primary sensorimotor cortex activation with taskperformance after fatiguing hand exercise," Exp. Brain Res. 167(2), 160-164 (2005).

7. Z. Jiang et al., "Strengthened functional connectivity in the brain during muscle fatigue," Neuroimage 60(1), 728-737 (2012).

8. J. Rhee and R. K. Mehta, "Functional connectivity during handgrip motor fatigue in older adults is obesity and sex-specific," Front. Hum. Neurosci. 12, 455 (2018).

9. T. Rupp et al., "Muscle, prefrontal, and motor cortex oxygenation profiles during prolonged fatiguing exercise," Adv. Exp. Med. Biol. 789, 149-155 (2013).

10. R. Thomas and P. Stephane, "Prefrontal cortex oxygenation and neuromuscular responses to exhaustive exercise," Eur. J. Appl. Physiol. 102(2), 153-163 (2008).

11. R. Radel, J. Brisswalter, and S. Perrey, "Saving mental effort to maintain physical effort: a shift of activity within the prefrontal cortex in anticipation of prolonged exercise," Cognit. Affective Behav. Neurosci. 17(2), 305-314 (2017)

12. J. W. Williamson, P. J. Fadel, and J. H. Mitchell, "New insights into central cardiovascular control during exercise in humans: a central command update," Exp. Physiol. 91(1), 51-58 (2006).

13. N. Naseer and K.-S. Hong, "fNIRS-based brain-computer interfaces: a review," Front. Hum. Neurosci. 9, 3 (2015).

14. T. J. Huppert et al., "HomER: a review of time-series analysis methods for near-infrared spectroscopy of the brain," Appl. Opt. 48(10), D280-D298 (2009).

15. N. S. Ward and R. S. Frackowiak, "Age-related changes in the neural correlates of motor performance," Brain 126(4), 873-888 (2003).

16. G. Derosiere and S. Perrey, "Relationship between submaximal handgrip muscle force and NIRS-measured motor cortical activation," Adv. Exp. Med. Biol. 737, 269-274 (2012).

17. J. Z. Liu et al., "Human brain activation during sustained and intermittent submaximal fatigue muscle contractions: an FMRI study," J. Neurophysiol. 90(1), 300-312 (2003).
18. J. Z. Liu et al., "Fatigue induced by intermittent maximal voluntary contractions is associated with significant losses in muscle output but limited reductions in functional MRI-measured brain activation level," Brain Res. 1040(1-2), 44-54 (2005).

19. R. C. Oldfield, "The assessment and analysis of handedness: the Edinburgh inventory," Neuropsychologia 9(1), 97-113 (1971).

20. K. M. McGregor et al., "Physical activity and neural correlates of aging: a combined TMS/fMRI study," Behav. Brain Res. 222(1), 158-168 (2011).

21. A. K. Singh et al., "Spatial registration of multichannel multi-subject fNIRS data to MNI space without MRI," NeuroImage 27(4), 842-851 (2005).

22. R. K. Mehta and A. E. Shortz, "Obesity-related differences in neural correlates of force control," Eur. J. Appl. Physiol. 114(1), 197-204 (2014).

23. J. Xu et al., "FC-NIRS: a functional connectivity analysis tool for nearinfrared spectroscopy data," Biomed. Res. Int. 2015, 1-11 (2015).

24. J. Cao et al., "Evaluation of cortical plasticity in children with cerebral palsy undergoing constraint-induced movement therapy based on functional near-infrared spectroscopy," J. Biomed. Opt. 20(4), 046009 (2015).

25. M. Kohl et al., "Determination of the wavelength dependence of the differential pathlength factor from near-infrared pulse signals," Phys. Med. Biol. 43(6), 1771-1782 (1998).

26. A. Yennu et al., "Prefrontal hemodynamic mapping by functional nearinfrared spectroscopy in response to thermal stimulations over three body sites," Neurophotonics 3(4), 045008 (2016).

27. A. Yennu et al., "Prefrontal responses to Stroop tasks in subjects with post-traumatic stress disorder assessed by functional near infrared spectroscopy," Sci. Rep. 6, 30157 (2016).

28. E. Visani et al., "Hemodynamic and EEG time-courses during unilateral hand movement in patients with cortical myoclonus. An EEG-fMRI and EEG-TD-fNIRS study," Brain Topogr. 28(6), 915-925 (2015).

29. A. R. Anwar et al., "Comparison of causality analysis on simultaneously measured fMRI and NIRS signals during motor tasks," in Conf. Proc. IEEE Eng. Med. Biol. Soc., pp. 2628-2631 (2013).

30. A. K. Singh and I. Dan, "Exploring the false discovery rate in multichannel NIRS," NeuroImage 33(2), 542-549 (2006).

31. M. Xia, J. Wang, and Y. He, "BrainNet viewer: a network visualization tool for human brain connectomics," PLoS One 8(7), e68910 (2013).

32. F. Tian, Z.-J. Lin, and H. Liu, "EasyTopo: a toolbox for rapid diffuse optical topography based on a standard template of brain atlas," Proc. SPIE 8578, 85782J (2013).

33. D. E. Hinkle, W. Wiersma, and S. G. Jurs, Applied Statistics for the Behavioral Sciences, 5th ed., Houghton Mifflin, Boston, Massachusetts (2002).

34. J. P. Marques, Applied Statistics Using SPSS, STATISTICA, MATLAB and $R$, Springer Publishing Company, Inc., London (2007).

35. D. H. Clarke, M. Q. Hunt, and C. O. Dotson, "Muscular strength and endurance as a function of age and activity level," Res. Q. Exercise Sport 63(3), 302-310 (1992).

36. G. C. Bogdanis, "Effects of physical activity and inactivity on muscle fatigue," Front. Physiol. 3, 142-142 (2012).

37. A. T. White et al., "Brain activation in multiple sclerosis: a BOLD fMRI study of the effects of fatiguing hand exercise," Mult. Scler. 15(5), 580-586 (2009).

38. Z. Jiang, X. Wang, and G. H. Yue, "Strengthened corticosubcortical functional connectivity during muscle fatigue," Neural Plast. 2016, 1-11 (2016).

39. A. W. Subudhi et al., "Frontal and motor cortex oxygenation during maximal exercise in normoxia and hypoxia," J. Appl. Physiol. 106(4), 1153-1158 (2009).

40. N. H. Kashou et al., "Hand-grasping and finger tapping induced similar functional near-infrared spectroscopy cortical responses," Neurophotonics 3(2), 025006 (2016).

41. R. S. J. Frackowiak et al., "Human brain function," Academic Press, San Diego, California (2004).

42. P. Wasson et al., "Predicting grip force amplitude involves circuits in the anterior basal ganglia," Neuroimage 49(4), 3230-3238 (2010).

43. S. Badoud et al., "Effects of dorsolateral prefrontal cortex lesion on motor habit and performance assessed with manual grasping and control of force in macaque monkeys," Brain Struct. Funct. 222(3), 1193-1206 (2017). 
44. L. H. Ernst et al., "Prefrontal activation patterns of automatic and regulated approach-avoidance reactions-a functional near-infrared spectroscopy (fNIRS) study," Cortex 49(1), 131-142 (2013).

45. M. Perrone-Bertolotti et al., "What is that little voice inside my head? Inner speech phenomenology, its role in cognitive performance, and its relation to self-monitoring," Behav. Brain Res. 261, 220-239 (2014).

46. M. Ypofanti et al., "Psychometric properties of the international personality item pool big-five personality questionnaire for the Greek population," Health Psychol. Res. 3(2), 2206 (2015).

47. J. Hardy, C. R. Hall, and L. Hardy, "Quantifying athlete self-talk," J. Sports Sci. 23(9), 905-917 (2005).

48. N. Zourbanos et al., "Automatic self-talk questionnaire for sports (ASTQS): development and preliminary validation of a measure identifying the structure of athletes' self-talk," The Sports Psychol. 23, 233-251 (2009).

49. A. F. T. Arnsten, "Stress signalling pathways that impair prefrontal cortex structure and function," Nat. Rev. Neurosci. 10(6), 410-422 (2009).

50. T.-W. Lin, S.-F. Tsai, and Y.-M. Kuo, "Physical exercise enhances neuroplasticity and delays Alzheimer's disease," Brain Plast. 4(1), 95-110 (2018).

51. T. B. Weng et al., "The acute effects of aerobic exercise on the functional connectivity of human brain networks," Brain Plast. 2(2), 171-190 (2017).

52. O. Seidel et al., "Motor learning in a complex balance task and associated neuroplasticity: a comparison between endurance athletes and nonathletes," J. Neurophysiol. 118(3), 1849-1860 (2017).

53. D. A. Raichlen et al., "Differences in resting state functional connectivity between young adult endurance athletes and healthy controls," Front. Hum. Neurosci. 10, 610 (2016).

54. C. P. Kaller et al., "Dissociable contributions of left and right dorsolateral prefrontal cortex in planning," Cereb. Cortex 21(2), 307-317 (2011).

55. H. Doi, S. Nishitani, and K. Shinohara, "NIRS as a tool for assaying emotional function in the prefrontal cortex," Front. Hum. Neurosci. 7, 770 (2013).

56. J. M. Spielberg et al., "A brain network instantiating approach and avoidance motivation," Psychophysiology 49(9), 1200-1214 (2012).

57. S. Perrey, "Promoting motor function by exercising the brain," Brain Sci. 3(1), 101-122 (2013).

58. T. Liu-Ambrose et al., "Resistance training and functional plasticity of the aging brain: a 12-month randomized controlled trial," Neurobiol. Aging 33(8), 1690-1698 (2012).

59. L. Gagnon et al., "Short separation channel location impacts the performance of short channel regression in NIRS," NeuroImage 59(3), 2518-2528 (2012).

60. M. D. Pfeifer, F. Scholkmann, and R. Labruyere, "Signal processing in functional near-infrared spectroscopy (fNIRS): methodological differences lead to different statistical results," Front. Hum. Neurosci. 11, 641 (2018).

61. I. Tachtsidis and F. Scholkmann, "False positives and false negatives in functional near-infrared spectroscopy: issues, challenges, and the way forward," Neurophotonics 3(3), 031405 (2016).
62. J. Cao et al., "Directional changes in information flow between human brain cortical regions after application of anodal transcranial direct current stimulation (tDCS) over Broca's area," Biomed. Opt. Express 9(11), 5296-5317 (2018).

Elizabeth L. Urquhart received her BS degree in biology from Furman University, Greenville, South Carolina. Currently, she is pursuing her $\mathrm{PhD}$ in bioengineering at the University of Texas at Arlington (UTA), under the tutelage of Dr. George Alexandrakis. Her research interests include functional near-infrared spectroscopy, brain imaging, and signal and image processing.

Hashini I. Wanniarachchi received her bachelor's degree in industrial and manufacturing systems engineering at UTA. Being passionate about technology and research, she is currently pursuing her $\mathrm{PhD}$ in biomedical engineering from UTA. Her research experience was in the field of near-infrared spectroscopy, electroencephalography, photobiomodulation, tissue optics, and signal and image processing.

Xinlong Wang received his BS degree in applied physics from Beijing University of Technology, China. He received his PhD through the BS to PhD program at UTA, where he received the "Excellent Graduates" award. Currently, he is a postdoctoral fellow for a joint project, involving UTA, UT Austin, and UT Southwestern Medical Center at Dallas, in the area of transcranial infrared photobiomodulation with multimode imaging and its applications.

Hanli Liu received her MS and $\mathrm{PhD}$ degrees in physics from Wake Forest University, followed by postdoctoral training at the University of Pennsylvania. She is a full professor of bioengineering and distinguished university professor at UTA. She is also a fellow of the American Institute for Medical and Biological Engineering. Her expertise lies in the field of near-infrared spectroscopy of tissues, functional optical brain imaging, transcranial photobiomodulation, and their clinical applications.

Paul J. Fadel received his MS degree in exercise physiology from Northeastern University and his PhD in integrative physiology from University of North Texas, followed by postdoctoral training at UT Southwestern Medical Center. He is now a full professor of kinesiology and director of Clinical Translational Science at UTA. His expertise lies in the field of investigation of neural control of the circulatory system and its applications at rest and during exercise in human health and disease.

George Alexandrakis did his undergraduate studies in physics at Oxford University, United Kingdom, and his graduate studies in medical physics at McMaster University, Canada. He was a postdoctoral fellow at Massachusetts General Hospital/Harvard Medical School and at UCLA. As faculty at the UTA Bioengineering Department, his research is focused on the development of optical imaging methods for biomedical applications. 